\title{
EDITORIAL
}

\section{Frailty and mental health: association with cognition, sleep, and well-being in older adults}

According to the Merriam-Webster dictionary, the use of the term frailty goes back to the $14^{\text {th }}$ century (in Merriam-Webster, n.d.). The Latin origin of the word comes from "fragilis", meaning liable to break. Today frailty may refer to physical disability or to a weakness of moral character. As Fried (2016) has pointed out eloquently, the unprecedented increase in average lifespan in the US from about 45 years in 1900 to 80 years today has added, essentially, a new stage of life. With increasing age, especially after age 70 , a new phenotype emerges, which is distinct from any single chronic disease and is an independent predictor of mortality in the short and intermediate term ( 3 and 7 years, respectively): the phenotype of frailty.

In 2013, a consensus group comprised of delegates from six major international, European, and American societies defined physical frailty as a medical syndrome with multiple causes and contributors that is characterized by diminished strength, endurance, and reduced physiological function that increases an individual's vulnerability for developing increased dependency and/or death (Morley et al., 2013). This group recommended that all persons older than 70 years and all individuals with significant weight loss $(\geq 5 \%)$ due to chronic disease should be screened for frailty, using simple, rapid, but validated screening tests such as the simple FRAIL scale (Morley et al., 2012).

Fried's criteria for frailty consist of a critical mass of three to five clinical components, including muscle weakness, slowed gait, low physical activity, sense of low energy or exhaustion, and unintentional weight loss (Fried, 2016). For most of the older adults who become frail, frailty is chronic and progressive. Those with a subclinical presentation of only one or two components have a 2.5-fold greater risk of progressing to full frailty phenotype within three years. The prevalence of frailty increases with age after age 65, and is twice as common in women as men and in African-Americans as compared to whites. By age 85 , one in four older adults in the community manifests the full frailty phenotype.

Much of the research literature on frailty has focused on physical health. However, mental health including cognition, sleep, social interactions, and positive aspects like well-being are as important as those related to physical diseases and disabilities.
This issue of International Psychogeriatrics includes three thoughtful and thought-provoking papers on mental health in relation to frailty (Duppen et al., Kaur et al., and Godin et al.) along with two commentaries (Rubtsova and Wennberg and St. Louis).

Optimal measurement of frailty is essential for both clinical and research purposes (Dent et al., 2016). Successful translation of frailty research into clinical practice requires the availability of standardized, validated, easy-to-administer tools, which address not only physical but also psychosocial aspects of frailty. The study by Duppen et al. (2019) exemplifies that direction. Drawing on the available theoretical conceptualizations of wellbeing as well as their qualitative studies, the authors developed a brief assessment of subjective wellbeing and validated it in a sample of older community-dwelling adults with or at risk of frailty. The Short Well-being Instrument for Older adults (SWIO) scale developed by these researchers includes nine items subdivided into three subscales: sense of mastery, meaning in life, and life satisfaction. Thanks to its brevity and ease of administration, SWIO is an excellent tool for use in various clinical settings as well as in research to evaluate well-being among older adults with or at risk of frailty. A laudable component of this work is its focus on a positive outcome like well-being among older adults, which is often missing in the frailty research in this population.

In her commentary, Rubtsova (2019) makes excellent suggestions to Duppen et al. She recommends further research to validate SWIO in clinical settings. She also encourages the investigators to take a broad perspective and think about potentially recursive relationship between frailty and wellbeing. Rubtsova's hypothesis that individuals with higher levels of well-being will be less likely to develop frailty; while transition to frailty may negatively affect well-being is sound and points to a need to experimentally test a resultant hypothesis that enhancing well-being would potentially diminish frailty or decrease the likelihood of adverse events. Rubtsova has urged the investigators to use SWIO to test these hypotheses in studies using a longitudinal design as well as intervention research. Future studies should indeed examine multifaceted frailty 
prevention interventions incorporating markers of psychosocial health and well-being.

Kaur and colleagues (2019) explored the associations between frailty, quality of sleep, and cognitive functioning, using a modified Fried frailty phenotype, the Pittsburgh Sleep Quality Index, and a standardized neuropsychological battery, respectively, in 154 community-dwelling and memory clinic older adults with memory complaints. The researchers found that frailty was directly associated with poorer executive function, but no other cognitive domains, and worse sleep quality, after adjusting for covariates and accounting for multiple comparisons. Poorer sleep quality was also associated with worse executive function and processing speed. Importantly, poor sleep mediated the relationship between frailty and executive function, processing speed, learning, and delayed recall - in other words, more frail individuals with poorer sleep quality showed worse performance in all those cognitive domains.

In their commentary, Wennberg and St. Louis (2019) emphasize the critical role of sleep for brain and general health, as sleep disturbances are highly prevalent among older adults, but they are also treatable. Insomnia and obstructive sleep apnea are especially common and remediable sleep disturbances, each affecting a third to a half of community-dwelling older adults. Cognitive behavioral therapy for insomnia (CBTI) is the mainstay for managing insomnia in adults. Data in older adult populations suggest that CBTI is more effective and safer than pharmacotherapy with hypnotic medications, which also carry a risk of falls and hip fractures. Diagnosis of obstructive sleep apnea requires polysomnography or a home sleep apnea test, followed in most cases by nasal continuous positive airway pressure therapy, which remains the most widely used treatment, though good patient outcomes depend on adherence to therapy. Adherence problems and tolerability issues are similar in older and younger populations. Other options include dental appliances and surgical alternatives. Restless legs syndrome and REM sleep behavior disorder respond well to specific medications.

Finally Godin et al. (2019) studied how cognitive impairment and frailty combine to impact older adults' Quality of Life (QoL). Using the data from the Survey of Health, Ageing, and Retirement in Europe, these investigators employed moderated regression, followed by simple slopes analysis. They found that worse levels of frailty and cognitive impairment were each associated with lower QoL. The strength of these relationships varied depending on interactions with age, sex, education, social vulnerability, and employment status. Higher social vulnerability was consistently associated with lower QoL. The authors appropriately note that, while health and cognitive function generally decline with age, several predictors of QoL are modifiable, providing potential targets to improve older adults' QoL. The relationships between health, cognition, and social circumstances that shape QoL in older adults are, however, complex, and point to a need for personalized interventions. I agree with Godin and colleagues that consideration of the complex interactions of social circumstances, including resources and vulnerabilities, is necessary to achieve a comprehensive understanding of QoL. A supportive social environment may have at least partially protective effect against declining QoL, despite cognitive impairment and frailty.

Measures of health-related QoL typically focus solely on the impact of physical and mental health. Godin and colleagues advocate for broadening the definition of QoL to include non-health related domains in order to offer a holistic approach for understanding how health can impact the QoL of older adults. As clarified by these authors, aspects of people's lives outside of health can impact QoL.

This study does have limitations. Like the Kaur et al. report, this one is cross-sectional, which means that causality or even directionality of associations cannot be inferred. Furthermore, the countries included in SHARE have varied social welfare and labor policies. Therefore, country-by-country analysis is necessary to assess the relationships among frailty, cognitive impairment, and QoL, especially for subgroups of people without employment or with disabilities. Future research may be able to surmise successful social and welfare policies. Two common limitations of most studies of older people are cohort and survivor biases. The cohort bias refers to the fact that the older adults were born and raised decades before the younger generations and were thus exposed to markedly different upbringing. Survivor bias points to the fact that the sickest people die young and do not reach old age. Longitudinal studies are, therefore, necessary to establish directionality as well as mediation and moderation effects of different variables on the outcomes.

One common criticism of mental health research is, however, not entirely accurate. It is often stated that mental health assessments mostly include selfreports, and therefore, are not valid - unlike objective measures like clinical measurements of biometrics and lab data. I believe that this critique is not quite accurate. Mental states are, by definition, subjective and cannot be evaluated by others. For example, perceived stress is more relevant to health than are objective markers of stress. Likewise, loneliness is subjective and does not necessarily owe its association with poor health to objectively measurable social isolation (Lee et al., 2018). 
There is some literature on frailty outside of geriatrics that also suggests an important role of mental health. In a study of young and middle-aged adults with versus without HIV infection, Rubtsova et al. (2018) found that frailty was associated with multiple psychosocial variables like grit, optimism, personal mastery, social support, emotional support. Factor analysis revealed that psychosocial variables loaded on two factors - positive resources/outlook and support by others. This suggests that positive resources and outlook may play a role in frailty prevention, although longitudinal studies are needed to establish causal links and then intervention studies to show efficacy of specific treatments.

Delaying, preventing, or treating frailty should be one of the healthcare priorities. Physical frailty can potentially be prevented or treated with certain interventions including physical exercise, proteincalorie supplementation, vitamin $\mathrm{D}$, and avoiding polypharmacy (Fried, 2016; Morley et al., 2013). There is a critical need for similar research focusing on psychosocial interventions to prevent and manage frailty. Studies are required to demonstrate that promoting resilience, optimism, self-efficacy, social engagement, and components of wisdom like emotional regulation reduce levels of stress, increase healthful behaviors, and improve lifestyle in older adults, thereby lowering the risk of frailty. That is positive psychiatry of aging (Jeste, 2018; Jeste and Lee, 2019).

\section{Funding}

This work was supported, in part, by the Sam and Rose Stein Institute for Research on Aging, University of California San Diego.

\section{Dilip V. Jeste ${ }^{1,2,3}$}

${ }^{1}$ Department of Psychiatry, University of California San Diego, La Jolla, CA, USA

${ }^{2}$ Sam and Rose Stein Institute for Research on Aging, University of California San Diego, La Jolla, CA, USA

${ }^{3}$ Department of Neurosciences, University of California San Diego, La Jolla, CA, USA

Email:djeste@ucsd.edu

\section{References}

Dent, E., Kowal, P. and Hoogendijk, E. O. (2016). Frailty measurement in research and clinical practice: a review. European Fournal of Internal Medicine, 31, 3-10.
Duppen, D., Rossi, G., Dierckx, E., Hoeyberghs, L. and De Donder, L. (2019). Focusing on positive outcomes in frailty research: development of a short well-being instrument for older adults (SWIO). International Psychogeriatrics, 31, 767-777.

Fragile [Def. 1]. (n.d.). In Merriam-Webster. Available at: https://www.merriam-webster.com/dictionary/ fragile\#learn-more; last accessed 10 June 2019.

Fried, L. P. (2016). Interventions for human frailty: physical activity as a model. Cold Spring Harbor Perspectives in Medicine, 6(6), a025916. doi: 10.1101/cshperspect. a025916.

Godin, J., Armstrong, J. J., Wallace, L., Rockwood, K. and Andrew, M. K. (2019). The impact of frailty and cognitive impairment on quality of life: employment and social context matter. International Psychogeriatrics, 31, 789-797.

Jeste, D. V. (2018). Positive psychiatry comes of age. International Psychogeriatrics, 30(12), 1735-1738.

Jeste, D. V. and Lee, E. E. (2019). Emerging empirical science of wisdom: definition, measurement, neurobiology, longevity, and interventions. Harvard Review of Psychiatry, 27(3), 127-140.

Kaur, S., Banerjee, N., Miranda, M., Slugh, M., SunSuslow, N.McInerney, K. F., Sun, X., Ramos, A. R., Rundek, T., Sacco, R. L. and Levin, B. E. (2019). Sleep quality mediates the relationship between frailty and cognitive dysfunction in non-demented middle aged to older adults. International Psychogeriatrics, 31, 779-788.

Lee, E. E., Depp, C., Palmer, B. W., Glorioso, D. K., Liu, J., Tu, X. M., Kim, H., Tarr, P., Yasunori, Y. and Jeste, D. V. (2018). High prevalence and adverse health effects of loneliness in community-dwelling adults across the lifespan: role of wisdom as a protective factor. International Psychogeriatrics. doi: 10.1017/ S1041610218002120.

Morley, J. E., Malmstrom, T. K. and Miller, D. K. (2012). A simple frailty questionnaire (FRAIL) predicts outcomes in middle aged African Americans. The fournal of Nutrition Health and Aging, 16, 601-608.

Morley, J. E., Vellas, B., van Kan, G. A., Anker, S. D., Bauer, J. M., Bernabei, R., Cesari, M. and Chumlea, W. C. (2013). Frailty consensus: a call to action. fournal of the American Medical Directors Association, 14(6), 392-397. doi: 10.1016/j.jamda.2013.03.022.

Rubtsova, A. A. (2019). Focusing on positive outcomes in frailty research: a step in a right direction. International Psychogeriatrics, 31, 759-761.

Rubtsova, A. A., Marquine, M. J., Depp, C., Holstade, M., Ellis, R. J., Letendre, D., Jeste, D. V. and

Moore, D. J. (2018). Psychosocial correlates of frailty among HIV-infected and HIV-uninfected adults. Behavioral Medicine. doi: 10.1080/08964289.2018. 1509053.

Wennberg, A. M. V. and St. Louis, E. K. (2019). Interconnectedness among frailty, sleep, and cognition: recent findings and clinical implications. International Psychogeriatrics, 31, 763-766. 